\title{
Gestão da Qualidade e dos riscos na segurança do paciente: estudo-piloto
}

\section{Sylvia Lemos Hinrichsen.}

Doutora e Mestre em Medicina Tropical pela Universidade Federal de Pernambuco (UFPE). Professora Adjunta da Universidade de Pernambuco(UPE) e Universidade Federal de Pernambuco(UFPE).

Email: sylviahinrichsen@hotmail.com

\section{Cristina Lúcia Ferraz de Oliveira.}

Graduada em enfermagem pela Faculdade de Enfermagem Nossa Senhora das Graças- UPE e MBA de Gestão em Controle de Infecção/INESP. Enfermeira do Controle de Infecção do Hospital Memorial São José. Email: oliveiraferrazcristina@ig.com.br

\section{Mislene de Albuquerque Campos}

Graduada em Engenheira Civil pela Universidade de Pernambuco. Pós-graduada em Engenharia de Segurança do Trabalho pela Universidade de Pernambuco. Engenheira de Segurança do Trabalho no Hospital Memorial São José.

Email: mislene.campos@hospitalmemorial.com.br

\section{Luana Clara de Melo Possas}

Graduada em Enfermagem pela Universidade de Pernambuco. Enfermeira do Controle de Infecção Hospitalar (CClH) - Hospital Memorial São José

E-mail: luapossas@hotmail.com

\section{Georgia Sabino.}

Graduada em Gestão Hospitalar. Supervisora de Qualidade no Hospital Memorial São José.

Email: georgia.sabino@gmail.com

\section{Tatiana de Aguiar Santos Vilella}

Farmacêutica e Biomédica. Mestrado em Medicina Tropical pela UFPE.

Farmacêutica do Hospital Naval de Recife. Analista Clínica do Laboratório Municipal de Saúde Pública do Recife.

Email: tatifarmaufpe@gmail.com.

RESUMO: É grande a preocupação das instituições de saúde com a segurança do paciente em todas as fases do cuidado assistencial e os seus possíveis riscos. O trabalho foi desenvolvido no período de janeiro/2009 a janeiro/2010 pela gerência de qualidade de um hospital privado, terciário, de alta complexidade pertencente à rede sentinela da Agência Nacional de Vigilância Sanitária em Recife, Brasil. Criaram-se grupos multidisciplinares para: identificar as ferramentas da cultura da qualidade e segurança do paciente; definir componentes da comissão de gerenciamento de riscos e desenhar o fluxograma da gestão de risco/qualidade. Foram definidas como fontes de notificação de riscos: colaboradores; lideranças; alertas de risco do serviço de atendimento ao cliente; comissão de controle infecções hospitalares; setor de gestão ambiental-saúde ocupacional; setor de gestão de riscos clínicos assistenciais; comissão de revisão de Óbitos/Prontuário e a farmácia. É fundamental sistematizar um processo educativo, permanente para as equipes multidisciplinares, focado nos riscos à saúde advindos de erros/eventos adversos/sentinela segundo legislações e padrões de qualidade.

PALAVRAS-CHAVE: Gestão do risco; qualidade; segurança do paciente.

\section{Quality management and risks in patient safety: Pilot Study.}

ABSTRACT: Today the health institutions are concerned about patient safety in all phases of care assistance and the possible risks. The work was carried out during the January/2009-January/2010 the quality management of a private hospital, tertiary, highly complex owned by sentinel network of the National Agency of Sanitary Surveillance in Recife, Brazil. Multidisciplinary teams were established to: identify the tools of the culture of quality and patient safety; define components of risk management committee and draw the flowchart of risk management/quality. Were defined as sources of risk reporting: employees, leaders, warnings customer service; hospital infections control committee; environmental management sector, occupational health sector risk management clinical care; deaths review committee/medical records and pharmacy. It is essential to systematize an ongoing education process for the multidisciplinary teams focused on risks to health arising from errors /adverse events/sentinel second laws and standards.

KEY-WORDS: Risk management; quality, patient safety. 


\section{INTRODUÇÃO}

Nos últimos anos tem sido grande a preocupação das instituições de saúde com a segurança do paciente em todas as fases do cuidado assistencial, especialmente pelos avanços tecnológicos e os seus possíveis riscos (LIMA et al.,2008; HARADA et al.,2006; PADILHA, 2001; PAINE et al.,2004; SANTOS et al., 2010; $\mathrm{KOHN}$ et al., 1999). Após a publicação do livro "To err is human" várias foram as discussões em torno da qualidade e segurança do paciente (KOHN et al., 1999; IHI, 2011; VRIES, 2008).

Eventos adversos ou também chamados de sentinela (os que causam danos permanentes e ou morte) ocorrem por falhas nos processos (atividades), na organização dos serviços (estrutura), na falta de liderança e ou de atitudes que modifiquem as realidades existentes (BRANCO FILHO, 2010; HINRICHSEN, 2010; LEAPE et al.,2009; BRASIL, 2007).

A incidência de eventos adversos nas instituições de saúde (hospitais) tem sido de um evento adverso para cada 10 internações hospitalares, sendo significativo o número de infecções relacionadas à assistência à saúde (IRAS)/ infecções hospitalares (IH) causando mortes (IHI, 2011; HINRICHSEN, 2010; ZAMBON, 2011; 0'BRIEN et al., 2011; COSTA et al, 2003).

Inúmeros são os fatores que contribuem para que existam erros na prática assistencial, uma vez que, ainda há uma interdependência entre os profissionais envolvidos, pois acreditam que estão fazendo a sua parte, e assim tudo fica na informalidade, sem um controle e sem processos sistematizados (LIMA et al.,2008; HARADA et al.,2006; PADILHA, 2001; PAINE et al.,2004).

Quando ocorre um erro, a primeira coisa que se busca é a culpa para que de imediato seja solucionado o problema, geralmente relacionada a uma punição que será a responsável pela não recorrência do erro. (ROSA, 2003).

Na maioria das instituições de saúde ainda não há a cultura de se procurar a falha no processo, na organização/estrutura e assim rever os possíveis planos de ação de reestruturação que venham a impedir novas falhas e ou eventos adversos, especialmente os do tipo sentinela que levam a dano permanente e ou morte (LIMA et al.,2008; HARADA et al.,2006; PADILHA, 2001).

Assim, para que se possam prevenir riscos, é necessário identificar e analisar a origem do evento para que ações preventivas possam ser sistematizadas de forma pró-ativa e não somente quando ocorrem. Estabelecer uma sistemática de gerenciamento de riscos na instituição poderá ser um caminho para se buscar um melhor controle e monitoramento de processos.

Uma cultura de qualidade é necessária como uma política institucional em todas as fases do cuidado do paciente segundo as diversas equipes multidisciplinares. No Brasil, ainda não se tem uma cultura de identificação/notificação de riscos e ou erros como oportunidades de melhorias, embora, em 2001, a Agência Nacional de Vigilância Sanitária (ANVISA) tenha criado o projeto dos hospitais sentinela com objetivos de identificar e monitorar eventos adversos através de uma rede de hospitais de referência (BRASIL, 2007).

No gerenciamento de riscos é fundamental que a instituição de saúde adote uma política de prevenção como um requisito para a qualidade, cujos principais objetivos sejam o de criar uma base mais sólida e segura para a tomada de decisão e para o planejamento da segurança do paciente, a partir do monitoramento e minimização e prevenção de incidentes que possam causar eventos adversos (IHI, 2011; BRANCO FILHO, 2010; HINRICHSEN, 2010). Este gerenciamento poderá ser feito por uma comissão multidisciplinar que sistematize processos que identifiquem todas as oportunidades e ameaças e que tire proveito das incertezas e variabilidades como aprendizados.

Dentro desse contexto, o presente trabalho foi desenvolvido com objetivo de desenho de um modelo para a gestão de risco na implantação de práticas assistenciais seguras para o paciente, segundo padrões internacionais de qualidade assistencial.

\section{METODOLOGIA}

O trabalho foi desenvolvido no período de janeiro de 2009 a janeiro 2010 pela gerência de qualidade/riscos de um hospital privado, terciário, de alta complexidade pertencente à rede sentinela da Agência Nacional de Vigilância Sanitária (ANVISA), em Recife, nordeste do Brasil. Foram criados grupos multidisciplinares para: 1 - identificar as ferramentas da implantação da cultura da qualidade e segurança do paciente; 2- definir os componentes de uma comissão multidisciplinar de gerenciamento de riscos/qualidade e 3- desenhar o diagrama (escopo) da gestão de risco e padrões de qualidade assistenciais a serem monitorados. 
A metodologia utilizada no estudo foi a de pesquisa ação já validada por algumas instituições de ensino e saúde cujos objetivos foram o de diagnosticar os processos assistenciais existentes e os seus impactos junto à qualidade/padrão de conformidade universal de uma política de qualidade sustentável (INFANTE; SANTOS, 2007).

Durante o período do estudo foram realizadas reuniões semanais (sempre no mesmo horário e dia da semana) com as principais lideranças das equipes multidisciplinares da instituição: Fase I - desenvolvida no período de janeiro/2009 a maio/2009 para o estudo do ambiente organizacional através da avaliação diagnóstica da instituição seguindo a metodologia pesquisa ação com foco nas principais fragilidades institucionais para possíveis adoecimentos decorrentes de riscos durante o processo assistencial multidisciplinar; Fase II - desenvolvida no período de junho/2009 a setembro/2009, onde foi discutida a fundamentação teórica da qualidade/principais definições sobre riscos e construídos modelos de processos relacionados ao cuidado segundo riscos que foram classificados em: sanitário, ambiental ocupacional e assistenciais. Nessa fase foram realizadas aulas expositivas, seminários, e-learning entre os membros das diversas equipes e estudos sistemáticos sobre riscos/qualidade/segurança do paciente; Fase III - desenvolvida no período de outubro/2009 a janeiro/2010, onde foram apresentados os desenhos dos processos para posterior implementação em pilotos e conseqüente validação pelas lideranças/alta gestão quanto ao escopo da gestão de riscos e segurança do paciente, segundo padrões internacionais de qualidade (Figura 1 e Quadro 1) (CBA, 2010; IQG, 2011).

\section{RESULTADOS}

Foram definidas como fontes de notificação de riscos (tipo passiva, ativa e sentinela): 1 - os colaboradores; 2- as lideranças; 3- os alertas de risco do SAC (Serviço de Atendimento ao Cliente); 4- a comissão de controle infecções hospitalares $(\mathrm{CClH})$; 5 - o setor de gestão Ambiental-saúde ocupacional ; 6 - o setor de gestão de riscos clínicos assistenciais; 7- a comissão de revisão de óbitos/prontuário; 8- a equipe de resposta rápida à pacientes críticos e 9- a farmácia.

Consideram-se como itens de classificação de segurança do paciente segundo a Organização Mundial de Saúde (ZAMBON, 2011): 1 - o tipo de evento (se evento adverso/quase falha/evento sentinela); 2- o tipo de desfecho do paciente (se dano: leve/moderado/grave/morte); 3- as características clínicas do paciente; 4- as características do evento notificado; 5- os desfechos ocorridos na Instituição; 6 - a detecção dos riscos; 7- os fatores de mitigação; 8- as ações de melhoria e 9- as ações preventivas de riscos adotadas sistematicamente em toda a instituição (Quadro 1).

Quadro 1- Definições da Cultura da Segurança do Paciente. Recife. 2009/2010.

\begin{tabular}{|l|l|}
\hline TERMOS & DEFINIÇÕES \\
\hline Eventos Adversos & $\begin{array}{l}\text { São incidentes não desejados, problemas } \\
\text { terapêuticos, danos iatrogênicos ou estabelecimento } \\
\text { de assistência à saúde que podem resultar de atos } \\
\text { deliberados ou de omissão }\end{array}$ \\
\hline Evento Sentinela & $\begin{array}{l}\text { Uma ocorrência inesperada envolvendo morte ou } \\
\text { dano grave, físico ou psicológico ou o risco advindo } \\
\text { desta. Dano grave refere-se, especificamente, a } \\
\text { perda de um órgão ou função. A expressão "ou o risco } \\
\text { advindo desta" inclui qualquer variação de processo } \\
\text { para o qual uma recorrência pode levar a uma chance } \\
\text { significativa de um evento adverso grave. }\end{array}$ \\
\hline Quase Falha & $\begin{array}{l}\text { Qualquer variação de processo que não afeta o } \\
\text { desfecho, mas cuja recorrência acarreta uma chance } \\
\text { significativa de um evento adverso grave. }\end{array}$ \\
\hline Queixa Técnica & $\begin{array}{l}\text { Qualquer suspeita de alteração, irregularidade, } \\
\text { mau funcionamento de um produto e/ou material } \\
\text { relacionado a aspectos técnicos ou legais e que poderá } \\
\text { ou não causar dano à saúde individual e/ou coletiva. }\end{array}$ \\
\hline
\end{tabular}




\begin{tabular}{|l|l|}
\hline TERMOS & DEFINIÇÕES \\
\hline Erro de Medicação & $\begin{array}{l}\text { Qualquer ato não intencional no processo de } \\
\text { prescrição, dispensa, transcrição ou administração } \\
\text { de uma droga ou medicamento. }\end{array}$ \\
\hline Condição de Risco & $\begin{array}{l}\text { Qualquer conjunto de circunstâncias (excluindo-se } \\
\text { os relacionados à doença para a qual o paciente está } \\
\text { sendo tratado), que aumenta significativamente a } \\
\text { probabilidade de um evento adverso grave. }\end{array}$ \\
\hline Erro de Processo/Ocorrência & $\begin{array}{l}\text { Um evento que não é consistente com a rotina de } \\
\text { cuidados ou procedimentos dohospital, podendo ou } \\
\text { não ter resultado em dano ou perda para um paciente, } \\
\text { familiar ou visitante, ou podendo levar a uma queixa } \\
\text { contra o hospital, um empregado ou um membro } \\
\text { da equipe médico-assistencial. São considerados } \\
\text { também erros de processos: erros de sistema, } \\
\text { falhas ou erros nos processos administrativos que } \\
\text { acarretem retrabalhos, custos adicionais ônus ou } \\
\text { prejuízo para a instituição. }\end{array}$ \\
\hline
\end{tabular}

Definiram-se como membros da comissão de gerenciamento de riscos-qualidade as lideranças de equipes multidisciplinares da instituição (Quadro 2).

Quadro 2- Componentes da Comissão de Gerenciamento de Riscos-Qualidade. Recife. 2009/2010.

\begin{tabular}{|l|l|}
\hline Componentes & Representantes \\
\hline Presidente & Diretor Médico/Clínico \\
\hline \multirow{3}{*}{ Membros Executores } & $\begin{array}{l}\text { Coordenador Técnico Geral da Gestão do Risco /Qualidade } \\
\text { Coordenador da Gestão do Risco Sanitário Hospitalar } \\
\text { Coordenador da Gestão do Risco Ambiental/Saúde Ocupacional } \\
\text { Coordenador da Gestão do Risco Clínico Assistencial }\end{array}$ \\
\hline \multirow{5}{*}{ Membros Consultores } & $\begin{array}{l}\text { Reprentante da Hotelaria/Infra estrutura } \\
\text { Representante da Farmácia } \\
\text { Representante da Agência Transfusional } \\
\text { Reprentante da Engenharia Clínica } \\
\text { Representante da Coordenação de Enfermagem } \\
\text { Representante da Educação Continuada/RH/Enfermagem } \\
\text { Representante de Enfermagem dos Blocos Cirúrgicos } \\
\text { Representante Médico das UTIs* } \\
\text { Representante Médico da Anestesia/Cirurgia } \\
\text { Representante da Comissão de Prontuário } \\
\text { Representante da Comunicação Externa/Midia/SAC* } \\
\text { Representante da CIPA* } \\
\text { Representante da Gestão de Processos } \\
\text { Representante do Processo de Qualidade/Enfermagem } \\
\text { Representante da Tecnologia da Informação }\end{array}$ \\
\hline
\end{tabular}

$\left.{ }^{*}\right)$ UTIs: Unidades de Terapia Intensiva; SAC: Serviço de Atendimento ao Cliente; CIPA: Comissão Interna de Prevenção de Acidentes. 
Quadro 3- Classificação dos Riscos. Recife. 2009/2010.

RISCOS CLÍNICOS
FOCO NO PACIENTE
É todo Risco associado à ação direta ou indireta
dos profissionais da área da saúde, resultante
da ausência / deficiência de políticas e ações
organizadas na prestação de cuidados de saúde.
Resulta quase sempre em eventos sentinelas, que
podem determinar danos irreversíveis a saúde
física ou psicológica dos pacientes.

\section{PADRÕES DE SEGURANÇA}

Acesso, Continuidade e Avaliação do cuidado Direitos e Educação do Paciente eFamiliares Anestesia e Cirurgia Uso de Medicamentos Metas Internacionais de Segurança do Paciente*

\section{RISCOS NÃO CLÍNICOS FOCO NA ADMINISTRAÇÃO}

\section{Estrutura Física}

Equipamentos Médicos

Ar condicionado

Riscos Elétricos e de Incêndio/Emergências

Gases Medicinais/Sistemas Utilitários (água -luz)

Higiene/Limpeza/Desinfecção/Esterilização

Insumos

Segurança Ocupacional/Segurança e Proteção

Gerenciamento de Resíduos/Materiais Perigosos

Qualidade e Segurança do Paciente

Prevenção e Controle de Infecções

Segurança das Instalações

Educação e Qualificação de Profissionais

Comunicação e Informação

Governo e Liderança

(*) Metas: 1 - Identificar os pacientes corretamente; 2- Melhorar a comunicação efetiva; 3- Melhorar a segurança dos medicamentos de alta vigilância; 4- Assegurar cirurgias em local de intervenção correto, procedimento correto e paciente correto; 5- Reduzir o risco de infecções associadas aos cuidados de saúde; 6- Reduzir o risco de lesões ao paciente decorrente de quedas.

\section{DISCUSSÃO}

A promoção de segurança e prevenção de danos deve ser a primeira consideração em todos os atos e tarefas, e é responsabilidade de todos os colaboradores, membros das equipes multidisciplinares, visitantes, familiares e pacientes (CBA, 2010; IQG, 2011; BRANCO, 2011; McCANNON et al., 2007).

A cultura de segurança e a contínua promoção de um ambiente seguro são adquiridas apenas através de capacitação, esforços coordenados e eficiente contribuição de cada indivíduo para o alcance deste objetivo através da pronta notificação de erros, eventos adversos e quase falhas, que permitirão a identificação e correção de problemas nos processos (BRANCO, 2011; McCANNON et al., 2007).

Para atingir notificação crescente, não deve ser enfatizado o "quem", mas o "como" dos erros e eventos adversos, ao mesmo tempo em que deve ser minimizada a culpabilidade individual (ROSA, 2003). E no processo de avaliação de erros e quase falhas os profissionais de saúde devem participar na notificação e no desenvolvimento de melhorias dos processos (KOHN et al., 1999; ROSA, 2003).

Os hospitais devem reconhecer que mesmo os profissionais competentes e cuidadosos estão sujeitos aos riscos nas suas atividades e que muitos erros são resultantes de sistemas inadequados e complexos (KOHN et al., 1999; ROSA, 2003; BRANCO, 2011). E erros e acidentes devem ser acompanhados na tentativa de estabelecer tendências e padrões para que se possa aprender com eles e prevenir sua recorrência, assim como aumentar a segurança dos pacientes (KOHN et al., 1999; ROSA, 2003).

A existência de um ambiente não punitivo e apoiador é fundamental para que se tenha a notificação das situações de riscos/erros e quase falhas, uma vez que, na sua maioria, os erros são, em geral, decorrentes do sistema e não só humanos (McCANNON et al., 2007).

Também é fundamental que seja estabelecida nas instituições de saúde um processo educativo permanente para todas as equipes multidisciplinares onde também sejam incluídos temas relativos aos erros/eventos adversos e melhoria contínua da segurança do cuidado do paciente segundo legislações e padrões de qualidade. 
Figura 1 - Diagrama da Gestão de Risco/Qualidade Institucional e Eventos Monitorados. Recife. 2009/2010.

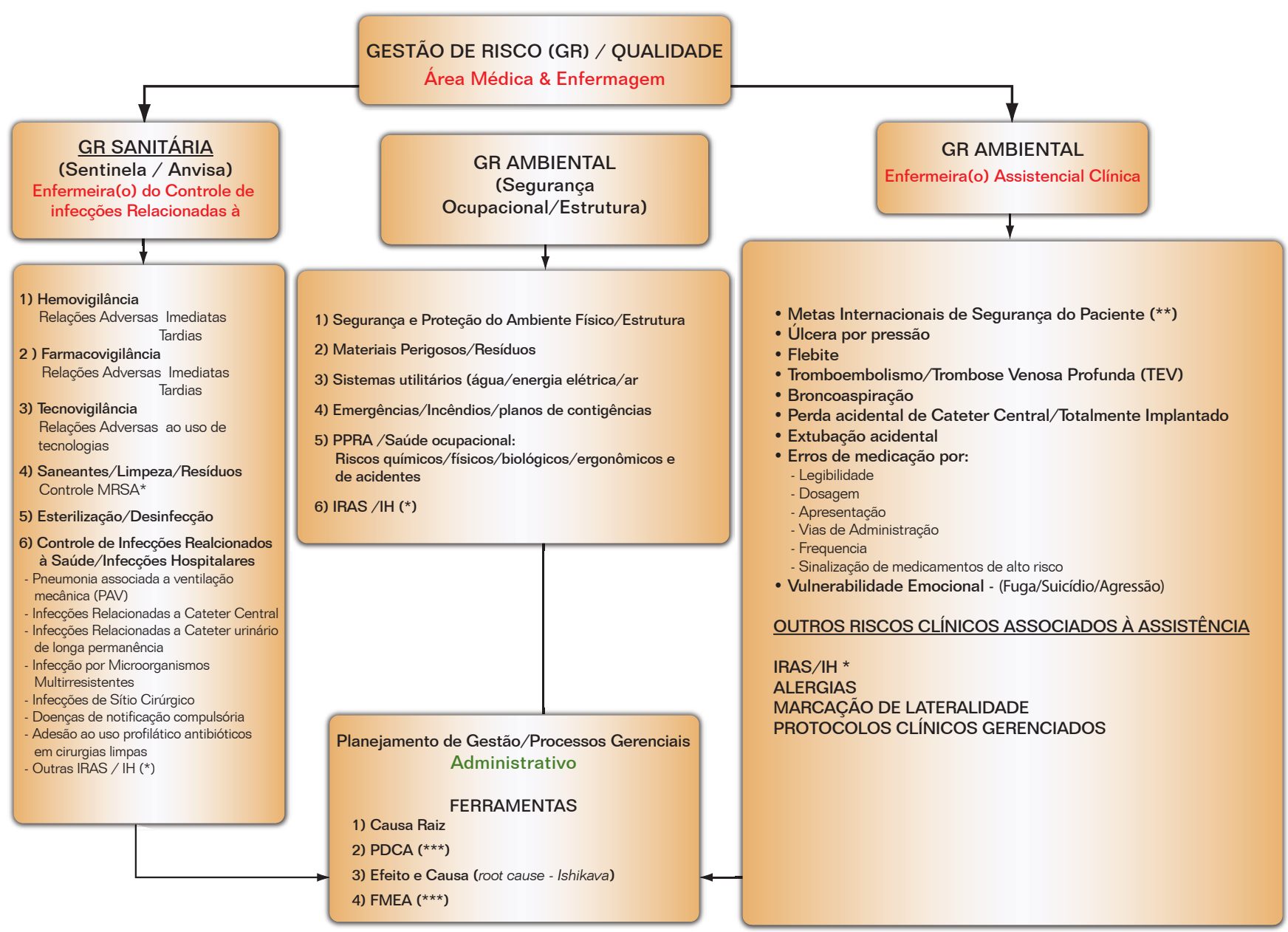

(*) MRSA: Staphylococcus aureus metilcilina resistente; IRAS / IR - Infecções Relacionadas à Assistência à Saúde/ Infecções Hospitalares.

(**) Metas - 1: Identificar os pacientes Corretamente; 2: Melhorar a Comunicação Efetiva; 3: Melhorar a Segurança de Medicamentos de AltaVigilância; 4: Assegurar Cirurgias com Local de Intervenção Correto, Procedimento Correto e Paciente Correto; 5: Reduzir o Risco de Infecções Associadas aos Cuidados de Saúde; 6: Reduzir o Risco de Lesões ao Paciente, decorrentes de Quedas. (***) 1- PDCA ( Plan/ DO/ Check/ Act); 2- FMEA ( Análise dos Efeitos e Modos Falha )

Através de uma política/diretriz institucional focada na gestão de riscos o hospital/instituição de saúde assume a responsabilidade pela melhoria contínua da qualidade dos serviços e dos cuidados de saúde prestados aos pacientes, promovendo, assim a redução das situações de riscos também para clientes, familiares, profissionais/ colaboradores, visitantes e meio ambiente.

A gestão de riscos (GR) do hospital passa então a contribuir na estruturação do sistema de informação relativo à identificação de toda a segurança não só do paciente, mas do ambiente onde ele está inserido durante o seu internamento. No que existe o respeito ao risco, assegura-se consequentemente a qualidade institucional (McCANNON et al., 2007).

Para que se tenha qualidade e segurança do paciente, um ponto de partida é a introspecção em todos os profissionais, pacientes, familiares e visitantes do pensamento estratégico baseado em riscos dentro da cultura organizacional (CBA, 2010).

No diagrama do gerenciamento de riscos deverão ser sistematizadas intervenções internas (erros, eventos adversos, eventos sentinela, \% de risco da doença e de tratamento) sempre baseadas em evidências científicas; assim como as intervenções externas (legislações) e as possíveis conseqüências (processos civis/por dano moral, imagem da instituição/profissional) advindas dos riscos (CBA, 2010; IQG, 2011; McCANNON et al., 2007).) 
A existência de um comitê/comissão de gestão de riscos formado por equipes multidisciplinares é fundamental para o monitoramento das potencialidades de eventos, assim como para fundamentar políticas, programas, fichas de coletas/fluxogramas, protocolos/ manuais/ material educativo e indicadores relativos a qualidade e segurança institucional (McCANNON et al., 2007; HINRICHSEN, 2008; ANAHP, 2011).

Através de auditorias internas, a equipe multidisciplinar poderá realizar atividades focadas na rastreabilidade dos riscos clínicos e não clínicos, observando as não conformidades existentes e os seus potenciais riscos á segurança do paciente e à qualidade institucional.

Observou-se no presente estudo o quanto é importante a existência da cultura de gestão de riscos, assim como da equipe multidisciplinar compondo uma comissão de monitoramento de situações adversas decorrentes das práticas assistenciais. Também é fundamental que haja a disseminação dos conceitos da cultura de segurança do paciente para que haja uma melhor introspecção desta em todas as fases de cuidados.

Criar um diagrama que identifique os riscos poderá ser uma ferramenta de cntrole da qualidade institucional. Nele não só se identificam as lideranças responsáveis pelo monitoramento das situações adversas, mas também os potenciais indicadores de controle da segurança do paciente.

Assim, sistematizar um processo educativo permanente para todas as equipes multidisciplinares focado em riscos (clínicos e não clínicos) à saúde advindos de erros/ eventos adversos/ sentinela, segundo legislações e padrões de qualidade poderá ser a melhor estratégia para se conseguir a qualidade institucional. Se diminuem riscos, aumenta-se a segurança e a qualidade institucional.

E nesse contexto a metodologia pesquisa-ação poderá ser uma importante ferramenta na introdução de conceitos e mudanças de realidades, pois através dela gera se na comunidade um processo auto diagnóstico e de autotransformação, pois considera o diálogo entre o saber científico e o saber individual na produção/construção de um novo conhecimento, permitindo ações e soluções de problemas coletivos, a partir da percepção dos envolvidos nos processos (INFANTE, 2007; THIOLLENT, 2005).

Existem várias definições para a palavra qualidade, mas todas têm como base as evidências de que se está seguindo as melhores práticas para se ter um resultado eficaz e eficiente. E, quando se vai a um hospital, não se espera que ele cause danos, embora estes possam ocorrer, daí a necessidade de gerenciá-los (HASSEN, 2011).

\section{REFERÊNCIAS}

AGÊNCIA NACIONAL DE VIGILÂNCIA SANITÁRIA- ANVISA (Brasil). Serviços de Saúde. Hospitais Sentinela [Internet]. 2007. Disponível em: <http://anvisa.gov.br/servicosaude/hsentinela/ index.htm >. Acesso em: 10 jan. 2011.

ASSOCIAÇÃO NACIONAL DE HOSPITAIS PRIVADOS (ANAHP). Gestão de Risco e Segurança evidenciam Governança Clínica nos hospitais da Anahp. Observatório ANAHP. São Paulo, p.12-17, 2011.

BRANCO FILHO, J.R.C. Tolerância zero em infecção hospitalar. Prática Hospitalar. v.69, p.34, 2010.

BRANCO FILHO, J.R.C. Construíndo um modelo de segurança do paciente. Prática Hospitalar. Ano XIII.n.74. Mar-Abr, p.8-9, 2010.

CONSÓRCIO BRASILEIRO DE ACREDITAÇÃO- CBA (Brasil). Padrões de Acreditação da Joint Commission International para Hospitais. Manual de Acreditação. 2010.pp.288.

COSTA, I.C.; HINRICHSEN, S.L.; ALVES, J.L et al. Prevalência e custos de processos infecciosos em unidade de terapia intensiva. Revista de Administração em Saúde. Vol. 5. n ${ }^{\circ}$ 20. p.7-16, 2003.

DE VRIES, E.N.; RAMRATTAN, M.A.; SMORENBURG, S.M et al. The incidence and nature of in -hospital adverse events: a systematic review. Quality \& safety in health care. v.17, n.3, p.21623, 2008. 
HARADA, M.J.C.S.; PEDREIRA, M.L.G.; PETERLINI, M.A.S.; PEREIRA, S.R. O erro humano e a segurança do paciente. São Paulo: Atheneu; 2006.

HASSEN, P. A meta é o dano zero. Melhores Práticas em Saúde, Qualidade e Acreditação. São Paulo, p.8-11, 2011.

HINRICHSEN, S.L. A ciência dos bundles e a segurança do paciente. Farmacêutico em Foco. n.1. Dezembro. p.10-11, 2010.

HINRICHSEN, S.L. Princípios da Administração de qualidade e o controle de infecções. Gerenciamento de riscos. Prática Hospitalar. v.60, p.57-63, 2008.

IHI. Institute of Healthcare Improvement - Campanha 5 Milhões de Vidas. Disponível em: < http:// www.ihi.org/IHI/Programs/Campaign/>. Acesso em: 30 set. 2011.

INFANTE, M.; SANTOS, M.A.B. A organização do abastecimento do hospital público a partir da cadeia produtiva: uma abordagem logística para a área de saúde. v.12, n.4, p.945-954, 2007.

INSTITUTO QUALISA DE GESTÃO. Accreditation Canada. Disponível em: > <www.iqg.com.br/ acreditaao-cchsa.php>.Acesso em: 20 nov. 2010.

KOHN, K.T; CORRIGAN, J.M.; DONALDSON, M.S(eds). To err is human: Building a safer healthsystem. Washington, DC: National Academy Press, 1999.

LEAPE, L.; BERWICK, D.; CLANCY, C.; CONAWAY, J. et al. for the Lucian Leape Institute at the National Patient Safety Foundation. Transforming healthcare: a safety imperative. Quality \& safety in health care. v.18, p.424-28, 2009.

LIMA, L.F.; LEVENTHAL, L.C.; FERNANDES, M.P.P. Identificando os riscos do paciente hospitalizado. Einstein. 2008; v.6, n.4, p.434-8.

MCCANNON, C.J; HACKBARTH, A.D; GRIFFIN, F.A. Miles to go: An introduction to the 5 Million Lives Campaign. Joint Commmission Journal on Quality and Patient Safety. v.33, n.80 p.477-484, 2007.

O'BRIEN, J.M.; LU, B.; ALI, N.A.; LEVINE, D.A.; et al. Insurance type and sepsis-associated hospitalizations and sepsis-associated mortality among US adults: a retrospective cohort study. Critical Care, 15:R130, 2011.

PADILHA, K.G. Ocorrências iatrogênicas na UTI e o enfoque de qualidade. Revista LatinoAmericana em Enfermagem, v.9, n.5, p.91-96, 2001.

PAINE, L.A.; BAKER, D.R.; ROSENSTEIN, B.; PRONOVOST, P.J. The Johns Hopkins Hospital: identifying and addressing risks and safety issues. Joint commission journal on quality and patient safety, v.30, n.10, p.543-550, 2004.

ROSA, M.B.; PERINI, E. Erros de medicação: quem foi? Revista da Associação Medica Brasileira. v.49, n.3, p.335-341, 2003.

SANTOS JUNIOR, B. J.; HINRICHSEN, S.L.;LIRA, C; VILELLA, T.A.S. Riscos Ocupacionais em centro de radiodiagnóstico. Revista de Enfermagem UERJ. Rio de Janeiro. v.18, n.3, p.365-70, 2010.

THIOLLENT, M. Perspectivas de Metodologia de Pesquisa Participativa e Pesquisa-ação na Elaboração de Projetos Sociais e Solidários. In: LIANZA, S. \& ADDOR, F. (orgs.). Tecnologia e Desenvolvimento Social e Solidário. Porto Alegre: Editora da UFRGS. 2005.

ZAMBOM, L.C. Classificação Internacional para a Segurança do Paciente da Organização Mundial de Saúde. Sistema de Resiliência. Disponível em:< http://www.medicinanet.com.br/ conteudos/gerenciamento/3018/classificacao_internacional_para_a_seguranca_do_paciente_ da_oms_\%E2\%80\%93_sistema_de_resiliencia.htm >. Acesso em: 12 fév. 2011. 